\title{
REDUCTION OF EULER'S EQUATIONS TO A CANONICAL FORM*
}

BY J. H. TAYLOR †

1. Introduction. As a by-product of the preparation of an earlier paper by the author, A generalization of Levi-Civita's parallelism and the Frenet formulas, Dissertation, University of Chicago, 1924, two useful methods of solving for the second derivatives in Euler's equations associated with the problem of minimizing an integral were discovered. In this paper these two methods are presented in detail. Whereas it appears at first that the assumptions required to effect the solution in the two instances are quite different, it is here shown that in each of the two cases the assumptions which are made may be replaced by the supposition that the $F_{1}$ function of the calculus of variations does not vanish.

2. The Euler Equations. Suppose that

$$
x^{\alpha}=x^{\alpha}(u), \quad u_{1} \leqq u \leqq u_{2}, \quad(\alpha=1, \cdots, n),
$$

are the equations of one of a class of curves joining two fixed points in an $n$-space, and let us consider the problem of selecting that curve of the class which gives to the integral

$$
I=\int_{u_{1}}^{u_{2}} F\left(x, x^{\prime}\right) d u
$$

its minimum value. Here $x$ and $x^{\prime}=d x / d u$ stand for the sets $x^{1}, \cdots, x^{n}$ and $x^{\prime 1}, \cdots, x^{\prime \prime}$ respectively. It will be assumed that $F$ satisfies the homogeneity condition

$$
F\left(x, x x^{\prime}\right)=\varkappa F\left(x, x^{\prime}\right),
$$

$\varkappa>0$.

* Presented to the Society, October 25, 1924.

$\uparrow$ National Research Fellow in Mathematics.

+ Transactrons of this Society, vol. 27 (1925). 
As a consequence of this it follows that

$$
F_{\alpha} x^{\prime \alpha}=F, \quad F_{\alpha \beta} x^{\prime \alpha}=0, \quad(\beta=1, \cdots, n),
$$

where the notation is $F_{\alpha}=\partial F / \partial x^{\prime \alpha}, F_{\alpha \beta}=\partial^{2} F / \partial x^{\prime \alpha} \partial x^{\prime \beta}$, and where we employ the convention that each index letter which appears twice in the same term is understood to indicate a summation with respect to that letter from 1 to $n$ as is customary in tensor analysis. From the second of equations (2) it follows that unless $x^{\prime}=0$, the determinant $\left|F_{\alpha \beta}\right|=0$.

The Euler differential equations defining the extremals for the integral $I$ are

$$
\begin{aligned}
\frac{d}{d u} F_{\beta}-\frac{\partial F}{\partial x^{\beta}}=F_{\beta c^{\prime}} x^{\prime \prime c}+\frac{\partial F_{\beta}}{\partial x^{\alpha}} x^{\prime \alpha}-\frac{\partial F}{\partial x^{\beta}}=0, \\
(\beta=1, \cdots, n) .
\end{aligned}
$$

These equations are not independent but satisfy the relation

$$
\left(F_{\beta \alpha^{\prime}} x^{\prime \prime \alpha}+\frac{\partial F_{\beta}}{\partial x^{\alpha}} x^{\prime \alpha}-\frac{\partial F}{\partial x^{\beta}}\right) x^{\prime \beta}=0,
$$

which is easily verified by differentiating the identity $F_{\beta} x^{\prime \beta}=F$ with respect to $u$ and applying (2). Let us suppose now that the parameter $u$ has been so chosen that along our solutions of equations (3) we have $F\left(x, x^{\prime}\right)=1$. Clearly such a selection is always possible if $F \neq 0$ along the solution, as we shall suppose, with the aid of the homogeneity condition (1). Since the determinant of coefficients $\left|F_{\alpha \beta}\right|$ vanishes we cannot solve (3) for the second derivatives by the usual process. Consider then the solution of the following system of equations

$$
\left\{\begin{aligned}
F_{\beta \alpha_{c} x^{\prime \prime}}+F_{\beta} w+\frac{\partial F_{\beta}}{\partial x^{\alpha}} x^{\prime \alpha}-\frac{\partial F}{\partial x^{\beta}} & =0 \\
F_{\alpha} x^{\prime \prime \alpha} & +\frac{\partial F}{\partial x^{\alpha}} x^{\prime \alpha}=0 .
\end{aligned}\right.
$$

It will be noticed that these differ from (3) in the introduction of an auxiliary variable $w$, and the adjoining of 
an equation obtained by differentiating $F=1$. We shall assume that the determinant of coefficients

$$
\left|\begin{array}{lllll}
F_{11} & F_{12} & \ldots & F_{1 n} & F_{1} \\
F_{21} & F_{22} & \cdots & F_{2 n} & F_{2} \\
\ldots \ldots & \ldots & \ldots & \ldots & \ldots \\
F_{n 1} & F_{n 2} & \cdots & F_{n n} & F_{n} \\
F_{1} & F_{2} & \cdots & F_{n} & 0
\end{array}\right|
$$

is not zero, and hence that the equations (5) may be solved for $x^{\prime \prime \prime}, w$ as functions of $\left(x, x^{\prime}\right)$. From (4) it follows at once that $w \equiv 0$, and therefore that the solutions $x^{\prime \prime c}$ of (5) are also solutions of (3).*

3. The F Function of the Calculus of Variations. If we suppose the rank of $\left|F_{\alpha \beta}\right|$ to be $n-1$, we have from the second of (2)

$$
\frac{x^{\prime 1}}{F^{\beta^{1}}}=\frac{x^{\prime 2}}{F^{\beta^{2}}}=\cdots=\frac{x^{\prime n}}{F^{\beta^{n}}}, \quad(\beta=1, \cdots, n),
$$

where $F^{\beta \alpha}$ denotes the cofactor of $F_{\beta \alpha}$ in $\left|F_{\mu \nu}\right|$. On multiplying hese ratios by $x^{\prime \beta}$ and making use of the symmetry

$$
F^{\alpha \beta}=F^{\beta \alpha}
$$

it is seen that we may define a function $F_{1}^{*}$ by the equations

or

$$
\frac{x^{\prime \beta} x^{\prime \alpha}}{F^{\beta \omega}}=\frac{1}{F_{1}^{*}}, \quad(\alpha, \beta \text { not summed })
$$

$$
x^{\prime \alpha} x^{\prime \beta} F_{1}^{*}=F^{\alpha \beta} \text {. }
$$

The usual notation for this function is $F_{1}$, but $F_{1}^{*}$ is used here to avoid confusion with $F_{1}=\partial F / \partial x^{\prime}$. From (7) it follows that

$$
F_{c} F_{\beta} x^{\prime c} x^{\prime \beta} F_{1}^{*}=F_{c} F_{\beta} F^{\alpha \beta},
$$

* This method of solution was suggested by an analogous scheme used by Mason and Bliss, The properties of curves in space which minimize a definite integral, Transactrons of this Society, vol. 9 (1908), p. 443. 
and, by (2), this becomes

Hence

$$
(F)^{2} F_{1}^{*}=F_{\alpha} F_{\beta} F^{\alpha \beta} .
$$

$$
F_{1}^{*}=\frac{-1}{(F)^{2}}\left|\begin{array}{lllll}
F_{11} & F_{12} & \cdots & F_{1 n} & F_{1} \\
F_{21} & F_{22} & \cdots & F_{2 n} & F_{2} \\
\cdots & \ldots & \ldots & \ldots & \ldots \\
F_{n 1} & F_{n 2}^{\prime} & \cdots & F_{n n} & F_{n} \\
F_{1} & F_{2} & \cdots & F_{n} & 0
\end{array}\right| .
$$

That is, the determinant (6) differs from the $F_{1}^{*}$ function by a non-zero factor.

4. A Second Method of Solution. Let a function $f\left(x, x^{\prime}\right)$ be introduced by

$$
f\left(x, x^{\prime}\right)=\frac{1}{2} F^{2}\left(x, x^{\prime}\right) .
$$

The homogeneity condition is now expressed by

$$
f\left(x, \varkappa x^{\prime}\right)=\varkappa^{2} f\left(x, x^{\prime}\right), \quad \boldsymbol{x}>0 .
$$

From (9) and (10) the following relations are readily deduced, the subscripts denoting as before the partial derivatives with respect to the corresponding $x^{\prime}$ variables :

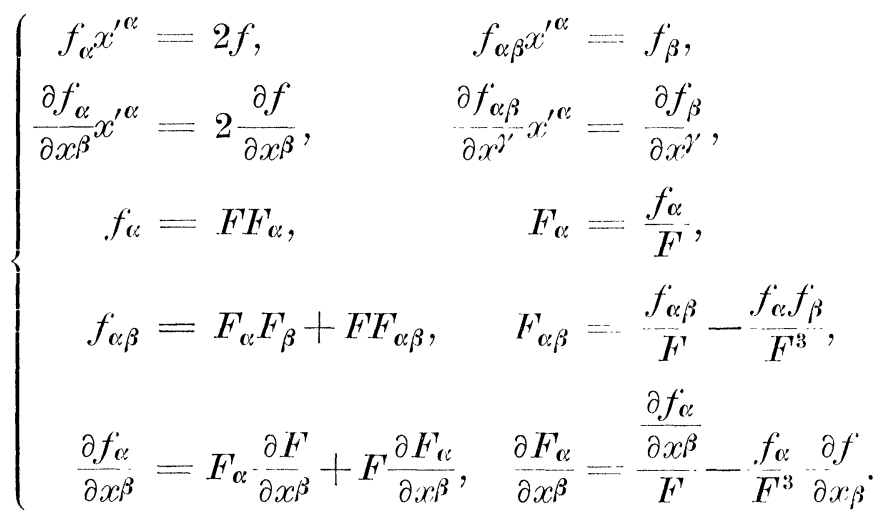

The Euler equations are

$$
F_{\beta} x^{\prime \prime \alpha}+\frac{\partial F_{\beta}^{\prime}}{\partial x^{\alpha}} x^{\prime \alpha}-\frac{\partial F^{\prime}}{\partial x^{\beta}}=0,
$$


or

$$
\frac{f_{\beta c^{\prime}} x^{\prime \prime \prime}+\frac{\partial f_{\beta}}{\partial x^{\alpha}} x^{\prime \prime \prime}-\frac{\partial f}{\partial x^{\beta}}}{F}=\frac{f_{\beta}}{F^{3}}\left(f_{c} x^{\prime \prime \prime}+\frac{\partial f}{\partial x^{\alpha}} x^{\prime c}\right) .
$$

If again we suppose the independent variable to be so selected that $F=1$, the right member reduces to zero and the equation becomes

$$
f_{\beta c c^{\prime \prime}} x^{\prime \prime \prime}+\frac{\partial f_{\beta}}{\partial x^{\prime \prime}} x^{\prime \prime}-\frac{\partial f}{\partial x^{\beta}}=0 .
$$

By (11), this may be written in the form

$$
f_{i c^{\prime}} x^{\prime \prime \prime}+\left(\frac{\partial f_{i \beta}}{\partial x^{\prime i}}-\frac{1}{2} \frac{\partial f^{\prime}}{\partial x^{i}}\right) x^{\prime{ }^{\prime \prime}} x^{\prime \beta^{3}}=0 .
$$

T'he question of the solution of these equations for the second derivatives forces our attention upon the determinant $\left|f_{\alpha \beta}\right|$.

The determinant in question is

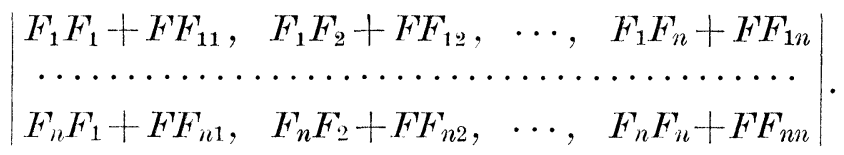

Since

$$
\left|F_{\alpha \beta}\right|=0
$$

the determinant reduces to

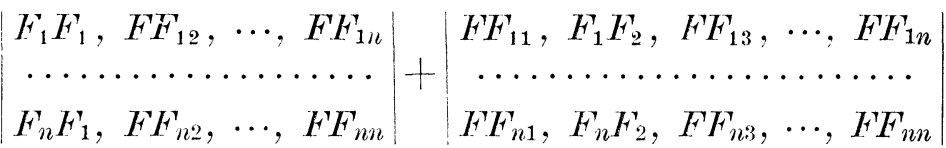

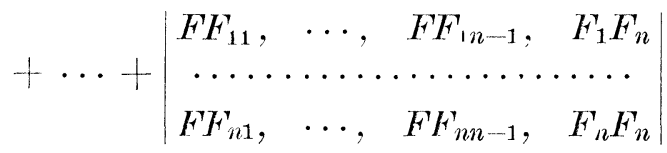

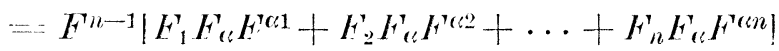

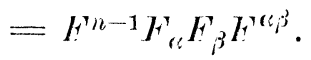


Hence

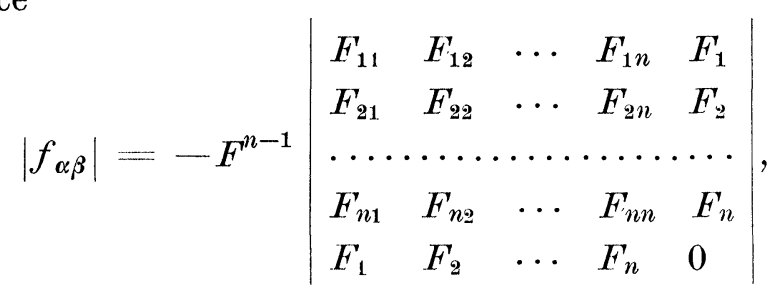

which is the same as the $F_{1}^{*}$ function except for a nonzero factor. If now we assume as before that the $F_{1}^{*}$ function is different from zero, and therefore $\left|f_{\alpha \beta}\right| \neq 0$, the solutions of (12) may be obtained in the form

$$
x^{\prime \prime \lambda}=-\Gamma_{\alpha \beta}^{\lambda} x^{\prime \alpha} x^{\prime \beta},
$$

where $\boldsymbol{I}_{\alpha \beta}^{\lambda}\left(x, x^{\prime}\right)$ is a function formed from $f_{\alpha \beta}\left(x, x^{\prime}\right)$ in precisely the same manner as the Christoffel symbol of the second kind $\{\alpha \beta, \lambda\}$ is formed from the quadratic form representing the square of the length element of a Riemann geometry. The explicit formulas for these coefficients are $\Gamma_{\alpha \beta}^{\lambda}\left(x, x^{\prime}\right)=f^{\lambda \mu}[\alpha \beta, \mu]$, where $f^{\lambda \mu}$ is the element of the reciprocal matrix of $f_{\alpha \beta}$ corresponding to the term $f_{\lambda, \mu}$, and

$$
[\alpha \beta, \mu]=\frac{1}{2}\left(\frac{\partial f_{\alpha \mu \mu}}{\partial x^{\beta}}+\frac{\partial f_{\beta \mu}}{\partial x^{\alpha}}-\frac{\partial f_{\alpha \beta}}{\partial x^{\mu}}\right) .
$$

The method of obtaining the solution of (12) in the form (13) is formally exactly the same as that used in obtaining the equations of the geodesics in a Riemann space.*

It may be remarked, in terms of the concepts of parallelism and curvature, as developed in the author's thesis mentioned in $\S 1$, that along a geodesic the tangent to the geodesic remains parallel to itself, or in other words that a geodesic is a curve of constant direction. A geodesic is also characterized as a curve of zero first curvature.

Princeton University

* See F. D. Murnaghan, Vector Analysis and the Theory of Relativity, 1922, pp. 89-90. 